lustitia Socialis. Revista Arbitrada de Ciencias Jurídicas.

Año V. Vol. V. N³. Edición Especial. 2020-III:

Universidad Regional Autónoma de los Andes

Hecho el depósito de Ley: FA2016000064 ISSN: 2542-3371

FUNDACIÓN KOINONIA (F.K). Santa Ana de Coro, Venezuela

Mónica del Rocío Mosquera-Endara; Andrés Sebastián Avalos-Salazar; Dany Alejandro Baquero-Marañón

http://dx.doi.org/10.35381/racii.v5i3.1095

\title{
Distribución de los carnets de discapacidad desde el ámbito administrativo en tiempos de pandemia
}

\section{Distribution of disability cards from the administrative sphere in times of pandemic}

\author{
Paul Centeno-Maldonado \\ ur.paulcenteno@uniandes.edu.ec \\ Universidad Regional Autónoma de los Andes, Riobamba \\ Ecuador \\ https://orcid.org/0000-0001-6541-8078 \\ Alex Dario Haro-Altamirano \\ alexdarioh10@gmail.com \\ Universidad Regional Autónoma de los Andes, Riobamba \\ Ecuador \\ Santiago Sebastian Peralta-Vargas \\ sebast 1993@yahoo.com \\ Universidad Regional Autónoma de los Andes, Riobamba \\ Ecuador
}

Recibido: 31 de octubre de 2020

Revisado: 10 de octubre de 2020

Aprobado: 05 de diciembre de 2020

Publicado: 10 de diciembre de 2020 
lustitia Socialis. Revista Arbitrada de Ciencias Jurídicas.

Año V. Vol. V. N³. Edición Especial. 2020-III:

Universidad Regional Autónoma de los Andes

Hecho el depósito de Ley: FA2016000064 ISSN: 2542-3371

FUNDACIÓN KOINONIA (F.K). Santa Ana de Coro, Venezuela

Mónica del Rocío Mosquera-Endara; Andrés Sebastián Avalos-Salazar; Dany Alejandro Baquero-Marañón

\title{
RESUMEN
}

Se tiene por objetivo analizar la distribución de los carnets de discapacidad desde el ámbito administrativo en tiempos de pandemia en Ecuador. Fundamentándose desde el enfoque cuantitativo, apoyada metodológicamente en un tipo descriptiva con diseño no experimental, lo cual permitió describir la realidad investigada tal como ocurre en la realidad, para lo cual se procedió a encuestar a 100 personas con discapacidad. La pandemia covid-19, trajo consigo un sinnúmero de actos de corrupción, poco a poco va desenmascarando a diario, bandas inescrupulosas que tanto daño causan al Ecuador, para combatir la corrupción hay que poner a recaudo todos estos actos para que no queden en la impunidad, puesto que las futuras generaciones tengan conocimiento de todos estos vergonzosos hechos.

Descriptores: Corrupción política; incapacidad; derecho civil. (Palabras tomadas del Tesauro UNESCO).

\begin{abstract}
The objective is to analyze the distribution of disability cards from the administrative sphere in times of pandemic in Ecuador. Based on the quantitative approach, methodologically supported by a descriptive type with non-experimental design, which allowed describing the reality under investigation as it occurs in reality, for which 100 people with disabilities were surveyed. The covid-19 pandemic brought with it countless acts of corruption, little by little it is unmasking on a daily basis, unscrupulous gangs that cause so much damage to Ecuador, to combat corruption all these acts must be collected so that they are not impunity, since future generations are aware of all these shameful events.
\end{abstract}

Descriptors: Political corruption; disabilities; civil law. (Words taken from the UNESCO Thesaurus). 
lustitia Socialis. Revista Arbitrada de Ciencias Jurídicas.

Año V. Vol. V. N³. Edición Especial. 2020-III:

Universidad Regional Autónoma de los Andes

Hecho el depósito de Ley: FA2016000064

ISSN: 2542-3371

FUNDACIÓN KOINONIA (F.K). Santa Ana de Coro, Venezuela

Mónica del Rocío Mosquera-Endara; Andrés Sebastián Avalos-Salazar; Dany Alejandro Baquero-Marañón

\section{INTRODUCCION}

En la actualidad, es un deber de todas y todos comprender desde esta perspectiva los derechos de las personas con discapacidad y respetarlos (Diniz, et al., 2009). También es deber del Estado garantizar el ejercicio de sus derechos a través de políticas públicas y medidas de acción afirmativa, sin exclusión alguna hacia este grupo vulnerable (Ocampo, 2018), en casos extremadamente excepcionales que exista algún tipo de actos de corrupción o ilegales que afecten a este grupo determinado, sancionar con todo el peso de la ley accesible para aquellos que piensan en beneficiarse de manera fraudulenta en la adquisición de las identificaciones de discapacidad que podrían favorecer a eludir los diferentes tipos de impuestos

Por lo tanto, la importancia de nuestra investigación se basa referentemente, en dar a conocer a los estudiantes de la carrera de Derecho de la Universidad Regional Autónoma de los Andes extensión Riobamba, la mala distribución de los carnets de discapacidad fraudulentos desde el ámbito administrativo en tiempos de pandemia COVID-19.

En este sentido, se tiene por objetivo analizar la distribución de los carnets de discapacidad desde el ámbito administrativo en tiempos de pandemia en Ecuador.

\section{METODO}

La investigación se fundamentó desde el enfoque cuantitativo, apoyada metodológicamente en un tipo descriptiva con diseño no experimental, lo cual permitió describir la realidad investigada tal como ocurre en la realidad, para lo cual se procedió a encuestar a 100 personas con discapacidad, mediante instrumento tipo cuestionario, el cual fue validado por juicio de expertos y cálculo de Alfa de Cronbach de 0,89 siendo confiable para su aplicación, lo cual permitió medir, primordialmente los tipos de discapacidad, grupos etarios, y perspectivas de los encuestados sobre el manejo del carnet de discapacidad, de ese modo, se analizó la información en razón de la estadística descriptiva para ser presentada en tabla y gráficos, mientras que para la parte del análisis documental se procedió a través de la técnica de análisis de contenido para identificar la 
lustitia Socialis. Revista Arbitrada de Ciencias Jurídicas.

Año V. Vol. V. №3. Edición Especial. 2020-III:

Universidad Regional Autónoma de los Andes

Hecho el depósito de Ley: FA2016000064

ISSN: 2542-3371

FUNDACIÓN KOINONIA (F.K). Santa Ana de Coro, Venezuela

Mónica del Rocío Mosquera-Endara; Andrés Sebastián Avalos-Salazar; Dany Alejandro Baquero-Marañón

información pertinente al tema.

\section{RESULTADOS}

Después de realizar por los análisis para determinar los tipos de discapacidad, género y grupos etarios se obtuvieron los siguientes resultados:

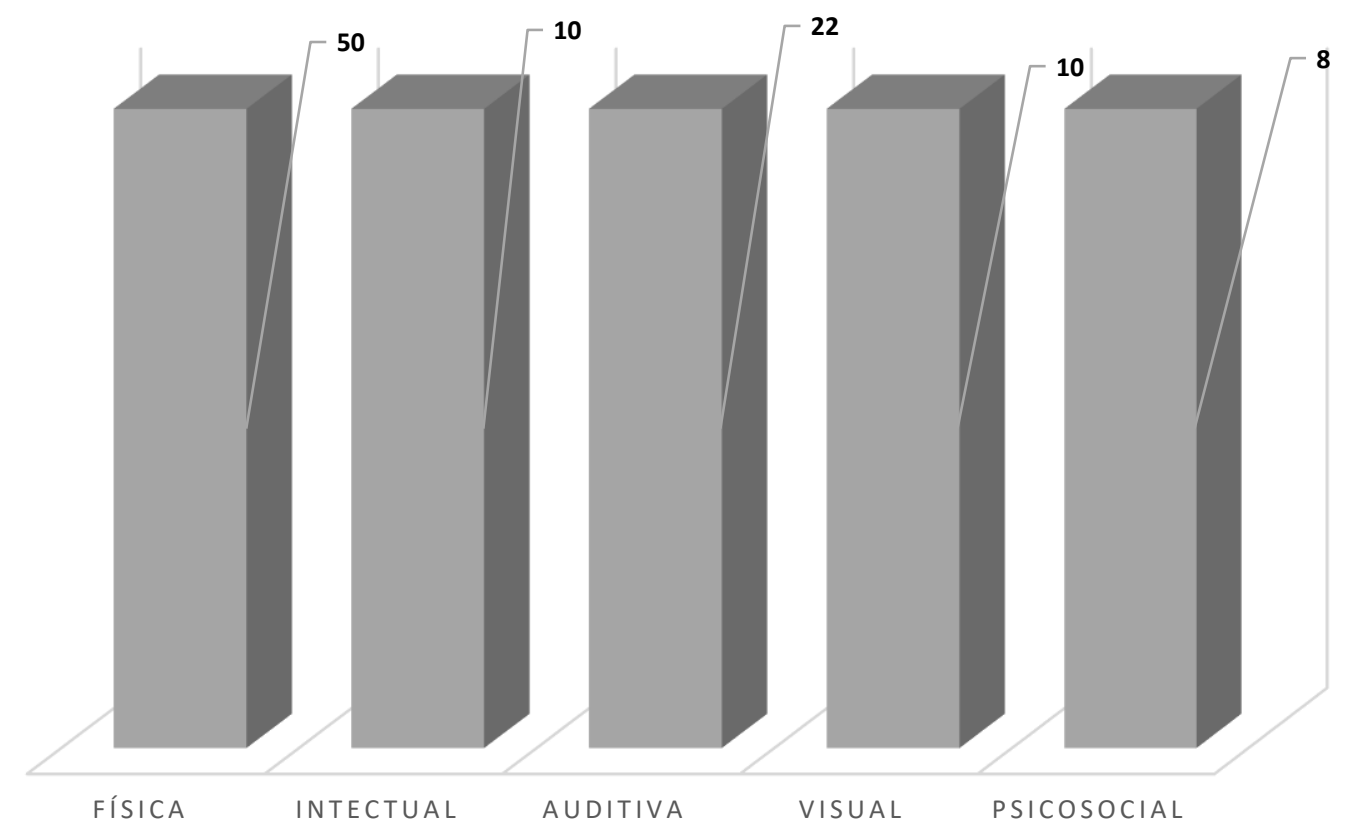

Grafico 1. Usuarios por tipo de discapacidad.

El $50 \%$ de los encuestados se relaciona con discapacidad física, el $10 \%$ con la intelectual, el $22 \%$ auditiva, $10 \%$ en discapacidad visual y el $8 \%$ en relación a la psicosocial.

Según los resultados adquiridos podemos darnos cuenta que existe mucha conmoción social últimamente el adquirir los carnés de discapacidad fraudulentos para acceder a derechos y beneficios que, por disposición de la Constitución de la República del Ecuador (2008), se deben otorgar a este denominado grupo vulnerable para poder equilibrar la balanza de oportunidades, por cuanto en muchas ocasiones han sido olvidados y 
lustitia Socialis. Revista Arbitrada de Ciencias Jurídicas.

Año V. Vol. V. N³. Edición Especial. 2020-III:

Universidad Regional Autónoma de los Andes

Hecho el depósito de Ley: FA2016000064

ISSN: 2542-3371

FUNDACIÓN KOINONIA (F.K). Santa Ana de Coro, Venezuela

Mónica del Rocío Mosquera-Endara; Andrés Sebastián Avalos-Salazar; Dany Alejandro Baquero-Marañón

excluidos por la sociedad por la situación crítica en la que viven a diario ya que a simple vista podemos darnos cuenta que en nuestra ciudad existen personas que verdaderamente lo necesitan (Arteaga, 2019).

Tras la llegada de la pandemia mundial Covid-19, en el Ecuador, a lo largo de estos cinco meses exactamente, se ha visto derrochado por un sinnúmero de actos de corrupción por parte de las autoridades en todos los ámbitos de dirección y manejo de esta terrible pandemia, solo basta con encender el televisor a diario para sumergirnos en una ola de noticias deplorables, que cada día enferman el entorno social; 2881 carnets de discapacidad fueron usados fraudulentamente según datos del ministerio de salud pública del Ecuador, es así que, en complemento, el presidente Lenin Moreno (2020), criticó a quienes utilizan o emplean el carnet para uso indebido como por ejemplo compra de vehículos a menor precio, ante lo cual, políticos ligados a la asamblea nacional, reaccionaron justificando su conducta al indicar que es una manera de alcanzar mejores precios.

El acto administrativo no tutela lo ilegal es decir que los carnets de discapacidad que fueron emitidos de manera fraudulenta, no se pueden usar con firmeza, y peor aún se puede hablar de legitimidad, de la que goza todo acto administrativo emitido de manera legal; la Ley Orgánica de Discapacidades establece como las formalidades más importantes para obtener la calificación de discapacidad: Una petición por parte de la persona interesada; y que en consecuencia la persona interesada tenga una discapacidad de al menos un 30\%; una vez que la persona cumpla con los diferentes requisitos solicitados podrá tener acceso hacia dicho requerimiento; que en muchas de las ocasiones personas de escasos recursos y que en verdad, necesitaban favorecerse de ellos, tenían que esperar meses, a ver si existía el pronunciamiento por parte de los encargados de emitir dichas peticiones.

Según los datos previos de la investigación realizada por varias fuentes, se presume que una red, de miembros del ministerio de salud pública son los que operaban y ofertaban, la adquisición de los carnets, en esos carnets se detectó que los exámenes o pruebas 
Mónica del Rocío Mosquera-Endara; Andrés Sebastián Avalos-Salazar; Dany Alejandro Baquero-Marañón

presentadas como justificativo médico, no se corresponde con la legalidad de los mismos, es decir, fueron falsificados, por lo que el COA establece que los administrados no pueden sufrir las consecuencias de los errores u omisiones de la Administración Pública, es decir los carnets se consideran nulos por lo que se ha comprobado, puesto que los actos administrativos pueden recaer sobre la objetividad real y no sobre supositorios fraudulentos, ya que la voluntad de la institución debe recaer en lo que plantea la ley y poder facilitar mediante este tipo de actos a personas que en realidad necesitan favorecerse por su condición de vulnerabilidad (Código Orgánico Administrativo COA)

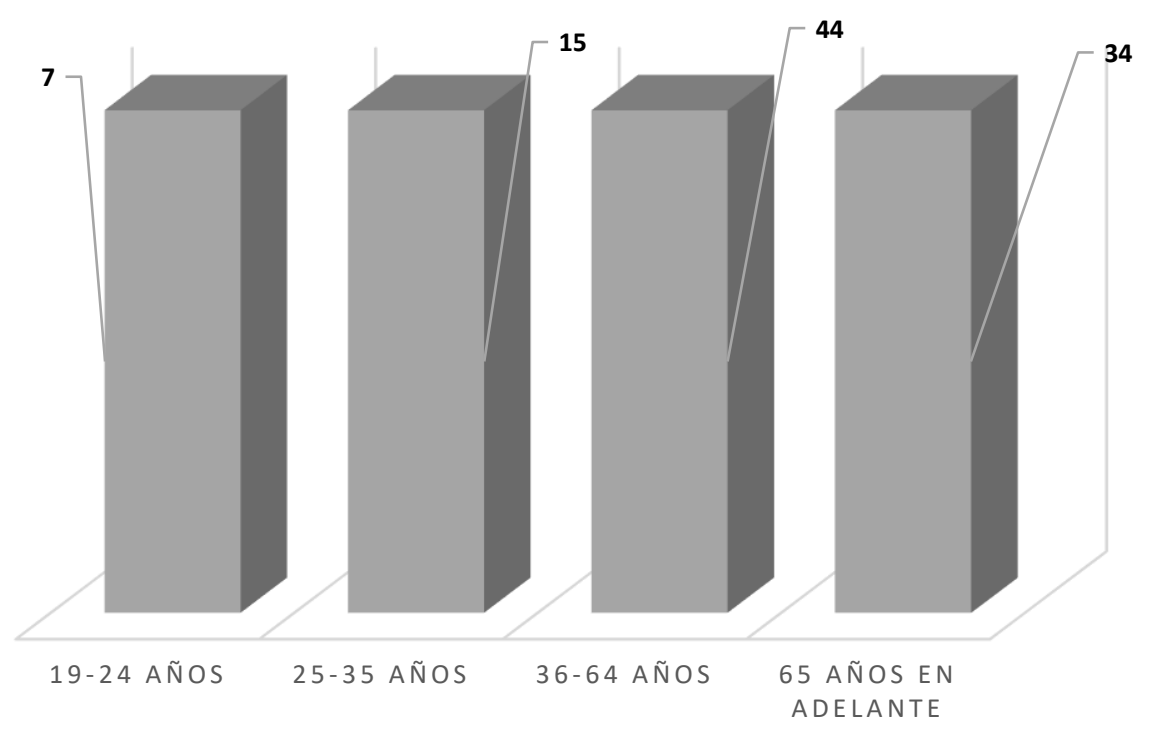

Grafico 2. Grupos etarios.

El grupo entre 19-24 años representa el 7\% de discapacitados encuestados, el 15\% representativo al segmento de 25-35 años, siendo la mayor concentración en la población de 36 -64 años con un 44\% y el 34\% restante, representado por los mayores de 65 años. Al analizar el rango de edad de las personas, existen diferentes tipos de discapacidades en el cual la Ley Orgánica de Discapacidades (LOD), constituye para que las personas 
lustitia Socialis. Revista Arbitrada de Ciencias Jurídicas.

Año V. Vol. V. N³. Edición Especial. 2020-III:

Universidad Regional Autónoma de los Andes

Hecho el depósito de Ley: FA2016000064

ISSN: 2542-3371

FUNDACIÓN KOINONIA (F.K). Santa Ana de Coro, Venezuela

Mónica del Rocío Mosquera-Endara; Andrés Sebastián Avalos-Salazar; Dany Alejandro Baquero-Marañón

con discapacidad, obtengan beneficios tributarios y aduaneros que cooperan para la importación de vehículos con exención de aranceles e impuestos, es decir en la exoneración de impuestos como el IVA e ICE en la importación de vehículos, el momento que se los declara nulos los actos mediante los cuales se otorgó los carnets a personas que efectivamente no padecían discapacidad alguna, es obligación de la autoridad recaudadora correspondiente redeterminar los impuestos declarados dentro del plazo de caducidad que le otorga la ley. Por lo que la declaratoria de nulidad de estos actos, no tiene efectos retroactivos (Art. $107 \mathrm{COA}$ ), siempre y cuando los terceros hayan adquirido estos beneficios de buena fe, generara efectos desde su expedición. (Ley Orgánica de Discapacidades, LOD).

En lo que va de 2020 las importaciones de autos de lujo suma 1.420 unidades; gracias a los beneficios adquiridos mediante este tipo de actividades ilegales, por personas que se encuentran bajo investigaciones previas, que aun la autoridad competente dentro de la investigación pueda determinar, es hora de que en nuestro país empiece a brillar la justicia sin distinción de raza o poder económico, ya que se ha evidenciado últimamente, que el poder judicial se encuentra enmascarado por actos de corrupción, ojala y este tipo de actos no queden en la impunidad, ya que se debe sancionar con todo el peso de la ley a este tipo de lacras que hoy en día van contaminando la sociedad, con sus atrocidades (El Universo, 2020).

Existió un pronunciamiento por parte de la Fundación Jóvenes Contra el Cáncer, exigiendo la destitución de las personas que, en el sector público o en el sector privado, hayan otorgado o se hayan beneficiado de manera fraudulenta de la obtención de estos carnets y que las personas con cáncer puedan acceder de manera temporal a estos documentos; no tiene lógica que personas que ganan más de $\$ 5000$ pidan exoneraciones, con patrimonios de miles de dólares. (El Universo, 2020b). 
lustitia Socialis. Revista Arbitrada de Ciencias Jurídicas.

Año V. Vol. V. N³. Edición Especial. 2020-III:

Universidad Regional Autónoma de los Andes

Hecho el depósito de Ley: FA2016000064

ISSN: 2542-3371

FUNDACIÓN KOINONIA (F.K). Santa Ana de Coro, Venezuela

Mónica del Rocío Mosquera-Endara; Andrés Sebastián Avalos-Salazar; Dany Alejandro Baquero-Marañón

\section{DISCUSIÓN}

El Ministerio de salud pública desplegará un nuevo software para el Sistema Nacional Electrónico de Discapacidades, que automatizará el proceso de calificación para evitar manipulaciones en la emisión de los carnés. Además, se suspenderán las claves de acceso al Sistema Informático de Línea (SIL), que sirve para emitir estos certificados y para asignar a cada persona su respectivo porcentaje de discapacidad, mientras se suscriben acuerdos de confidencialidad con cada funcionario del equipo de calificación para la entrega del documento.

Además, el Ministerio señalo que presentará las respectivas denuncias en la Fiscalía en contra de funcionarios y exfuncionarios de la institución que se encuentran involucrados en estos presuntos hechos de corrupción. El Ministerio revisará todos los carnés emitidos durante la emergencia sanitaria por covid-19 y sancionará a los servidores que hayan incumplido con las normativas y sus obligaciones propias, enmarcadas dentro de estos imperdonables actos de corrupción (Zevallos, 2020).

En confianza de que se puedan adoptar las medidas oportunas, para combatir todo tipo de corrupción, que va matando lentamente las aspiraciones futuras de nuestro país y para los que vienen, sean castigados estas ratas con todo el peso de la ley, en consecuencia, del daño irreparable que causaron con sus actuaciones erróneas.

La Ley Orgánica de Discapacidades refiere que,

...si una persona con discapacidad quiere comprar un vehículo que sea exclusivamente para mejorar su transporte se beneficiará de una rebaja de 8 mil dólares del costo del vehículo y luego de evaluaciones también podría acceder a una disminución del $50 \%$ en el precio final.

Según los datos previos de la investigación realizada, se presume que una red, de miembros del ministerio de salud pública son los que operaban y ofertaban, la adquisición de los carnets, se detectó que los exámenes de los especialistas no validan la condición de discapacidad de los portadores, adjuntan certificados emitidos por un mismo profesional con diversos diagnósticos, hojas en blanco o documentos sin firmas y sellos, 
lustitia Socialis. Revista Arbitrada de Ciencias Jurídicas.

Año V. Vol. V. N³. Edición Especial. 2020-III:

Universidad Regional Autónoma de los Andes

Hecho el depósito de Ley: FA2016000064

ISSN: 2542-3371

FUNDACIÓN KOINONIA (F.K). Santa Ana de Coro, Venezuela

Mónica del Rocío Mosquera-Endara; Andrés Sebastián Avalos-Salazar; Dany Alejandro Baquero-Marañón

por otro lado, se establece que "unos 2.454 carnés de discapacidad están en proceso de anulación y 22 funcionarios son investigados por la entrega fraudulenta de estos documentos" (Tapia, 2020), asegurando además que en lo que va de "2020 las importaciones de autos de lujo suma 1.420 unidades; gracias a los beneficios adquiridos mediante este tipo de actividades ilegales".

Para que una persona pueda adquirir el carnet de discapacidad, debe determinar de manera específica el problema o situación de salud vulnerable, inicialmente debe recurrir a una cita médica en un hospital o centro de salud público. Donde un galeno especializado, mediante revisión evalúa el porcentaje de discapacidad que tenga la persona que puede ser auditiva, física, intelectual, de lenguaje, psicosocial o visual.

\section{CONCLUSIONES}

La pandemia covid-19, trajo consigo un sinnúmero de actos de corrupción, poco a poco va desenmascarando a diario, bandas inescrupulosas que tanto daño causan al Ecuador, para combatir la corrupción hay que poner a recaudo todos estos actos para que no queden en la impunidad, puesto que las futuras generaciones tengan conocimiento de todos estos vergonzosos hechos.

Se ha evidenciado la falta de oportunidades que tienen las personas con algún grado de discapacidad, para poder tener una ayuda, referente a testimonios verdaderos, relatan que han tenido que equiparar una ardua lucha a diario, pasando de oficina en oficina, recibiendo a diario tratos prepotentes y hasta valoraciones inmediatas en son de burla haciendo referencia que no son dignos de recibir dicha documentación.

Esperamos que las autoridades competentes que se encuentran conociendo dicha problemática, puedan actuar de una manera arbitraria, y que se castigue con todo el peso de la ley a estos "personajes", que van contaminando el buen nombre de las diferentes dependencias del estado, con sus actitudes nefastas. 
lustitia Socialis. Revista Arbitrada de Ciencias Jurídicas.

Año V. Vol. V. N³. Edición Especial. 2020-III:

Universidad Regional Autónoma de los Andes

Hecho el depósito de Ley: FA2016000064 ISSN: 2542-3371

FUNDACIÓN KOINONIA (F.K). Santa Ana de Coro, Venezuela

Mónica del Rocío Mosquera-Endara; Andrés Sebastián Avalos-Salazar; Dany Alejandro Baquero-Marañón

\section{FINANCIAMIENTO}

No monetario.

\section{AGRADECIMIENTO}

A la Universidad Regional Autónoma de los Andes; por motivar el desarrollo de la Investigación.

\section{REFERENCIAS CONSULTADAS}

Arteaga, A. (2019). Inclusión laboral de las personas con discapacidad. [Labor inclusion of people with disabilities]. IUSTITIA SOCIALIS, 4(1), 16-38. http://dx.doi.org/10.35381/racii.v4i1.536

Código Orgánico Administrativo COA. Segundo Suplemento del Registro Oficial No.31, 7 de Julio 2017. Recuperado de https://n9.cl/2v4j5

Diniz, D, Barbosa, L, \& Santos, dos-Wederson R. (2009). Discapacidad, derechos humanos y justicia. [Disability, human rights and justice]. Sur. Revista Internacional de Direitos Humanos, 6(11), 64-77. https://doi.org/10.1590/S1806$\underline{64452009000200004}$

El Universo (2020). Importación de vehículos para personas con discapacidad se triplicó desde el 2017. [Import of vehicles for people with disabilities tripled since 2017]. Recuperado de https://n9.cl/ne4z

EI Universo (2020b). Iniciativas ciudadanas para investigar irregularidades en carnés de discapacidad. [Citizen initiatives to investigate irregularities in disability cards]. Recuperado de https://n9.cl/1ijlx

Ley Orgánica de Discapacidades. Suplemento Registro Oficial № 796. Martes 25 de septiembre del 2012 - 3. Recuperado de https://n9.cl/zxfi

Moreno, L. (2020). Presidente Lenin Moreno ordena al Ministerio de Salud investigar la entrega de carnés de discapacidad a asambleístas. [President Lenin Moreno orders the Ministry of Health to investigate the delivery of disability cards to assembly members]. Recuperado de https://n9.cl/zgmts 
lustitia Socialis. Revista Arbitrada de Ciencias Jurídicas.

Año V. Vol. V. N³. Edición Especial. 2020-III:

Universidad Regional Autónoma de los Andes

Hecho el depósito de Ley: FA2016000064 ISSN: 2542-3371

FUNDACIÓN KOINONIA (F.K). Santa Ana de Coro, Venezuela

Mónica del Rocío Mosquera-Endara; Andrés Sebastián Avalos-Salazar; Dany Alejandro Baquero-Marañón

Ocampo, J. (2018). Discapacidad, Inclusión y Educación Superior en Ecuador: El Caso de la Universidad Católica de Santiago de Guayaquil. [Disability, Inclusiveness and Higher Education in Ecuador: The Case of Universidad Católica de Santiago de Guayaquil]. Revista latinoamericana de educación inclusiva, 12(2), 97114. https://dx.doi.org/10.4067/S0718-73782018000200097

Tapia, L. (2020). 22 funcionarios son investigados por entrega fraudulenta de carné de discapacidad. [22 officials are investigated for fraudulent delivery of disability cards]. Recuperado de https://n9.cl/iifi

Zevallos, J. C. (2020). 3000 carnés de discapacidad se emitieron de 'manera ilegal', durante la pandemia del covid-19. [3 000 disability cards were issued 'illegally', during the covid-19 pandemic]. Recuperado de https://n9.cl/loyp4

C2020 por los autores. Este artículo es de acceso abierto y distribuido según los términos y condiciones de la licencia Creative Commons Atribución-NoComercial-Compartirlgual 4.0 Internacional (CC BY-NC-SA 4.0)

(https://creativecommons.org/licenses/by-nc-sa/4.0/). 